Tomás-Folch, M. \& Duran-Bellonch, M. (2017). Comprendiendo los factores que afectan la transferencia de la formación permanente del profesorado. Propuestas de mejora. Revista Electrónica Interuniversitaria de Formación del Profesorado, 20 (1), 145-157.

\title{
Comprendiendo los factores que afectan la transferencia de la formación permanente del profesorado. Propuestas de mejora
}

\author{
Marina Tomàs-Folch, y Mar Duran-Bellonch \\ Universitat Autònoma de Barcelona
}

\section{Resumen}

En este artículo se aborda la transferencia de la formación docente en la universidad.

Se presentan los resultados de indagar cualitativamente en la comprensión de los factores del modelo Holton, Bates y Ruona (2000) y de solicitar a personas expertas propuestas para incrementar la transferencia.

Se llevan a cabo 3 entrevistas grupales (focus group) en las 3 universidades participantes en el estudio, sobre la determinación de los factores para evaluar la transferencia de la formación docente en la universidad. En los grupos, compuestos por 21 personas con responsabilidades en la formación docente universitaria, se ahondó en los resultados cuantitativos obtenidos sobre los factores que afectan a la transferencia del aprendizaje.

Se confirma la relevancia de los factores identificados en el cuestionario: diseño de la formación y aprendizaje realizado; apoyo del responsable docente; predisposición al cambio; recursos del entorno; feedback del estudiante; reconocimiento institucional; cultura docente del equipo de trabajo y organización personal del trabajo.Además, expertos y expertas aportan propuestas para incrementar la transferencia, confirmando y ampliando el significado de los factores que la facilitan o dificultan, relacionadas con lo personal, lo relativo al programa formativo y lo organizativo o cultural.

\section{Palabras clave}

Transferencia; profesorado universitario; formación docente; desarrollo profesional.

\section{Contacto:}

Marina Tomàs-Folch, marina.tomas@uab.cat, Facultad de Ciencias de la Educación, UAB Edificio G6, despacho 26808193 Cerdanyola del Vallès (Barcelona)

Este artículo está vinculado al Grupo REDU sobre "¿Cómo medir la transferencia de la formación en Educación Superior? Herramienta y resultados"formado por Mònica Feixas (Coord.)M. Mar Duran , Idoia Fernández, Amparo Fernández, M. José García San Pedro, M. Dolors Márquez, Pilar Pineda , Carla Quesada, Sarai Sabaté ,Marina Tomàs-Folch, Franziska Zellweger Moser y Patricio Lagos (colaborador) 


\title{
Understanding the factors affecting transfer of university teachers' permanent training. Proposals for improvement
}

\begin{abstract}
This article addresses the transfer of teacher training in the university.

In this article, we present the results of the qualitative inquiry to comprehend these factors of Holton, Bates, and Ruona (2000) and of requesting experts to offer proposals to increase transfer.

Three focus groups were carried out in the three participating universities of the study to determine the factors to employ for the assessment of university transfer teacher's training. The groups comprised 21 people in charge of university teacher training. The quantitative results obtained regarding the factors affecting learning transfer were examined in depth.

We confirmed the relevance of the factors identified in the questionnaire: the design of the training and learning carried out; the support of the teacher in charge; predisposition to change; resources of the setting; student feedback; institutional acknowledgment; teaching culture of the work team and personal organization of the work. In addition, the experts contribute proposals to increase transfer, confirming and extending the meaning of the factors that facilitate or hinder transfer related to personal aspects, the training program, and organizational or cultural aspects.
\end{abstract}

\section{Key words}

Transfer; teacher's training; university teachers; teaching development.

\section{Introducción}

La formación docente en la Educación Superior debería dar lugar a un cambio en las prácticas docentes, lo que a su vez, debería influir positivamente en el aprendizaje del estudiante, pero lo segundo no es posible sin lo primero. El personal docente, cuando ya se ha formado, tiene que aplicar lo aprendido en el aula, o lo que es lo mismo, ha de transferir su aprendizaje. Sólo así se producirán cambios en su praxis docente, que son los que han de facilitar que mejore el proceso de aprendizaje del estudiantado. La investigación realizada pretende contribuir a la comprensión de los factores que condicionan esta transferencia de la formación a la práctica docente en el aula.

En este artículo presentamos los resultados obtenidos de llevar a cabo 3 entrevistas grupales (focus group) en las 3 universidades participantes en el estudio.

Los grupos estaban compuestos por personas con responsabilidades en la formación docente universitaria, que ahondaron en los porqués de los resultados cuantitativos obtenidos sobre los factores que afectan a la transferencia del aprendizaje, hallados mediante proceso cuantitativo en la investigación. Resulta de especial interés los relativos a dos de los factores condicionantes de la transferencia según los resultados de Feixas, Fernández,Lagos, Quesaday Sabaté(2013 b).

Además de contrastar los resultados obtenidos sobre los factores facilitadores y/o obstaculizadores de la aplicación de lo aprendido, las entrevistas grupales también sirvieron 
para recoger las ideas y propuestas de los expertos en procesos de formación docente en las universidades, sobre cómo incrementar la transferencia de la formación impartida al profesorado universitario.

\section{La transferencia de la formación docente universitaria}

Evaluar los resultados obtenidos mediante procesos de formación continua es una necesidad aceptada por todas las organizaciones que invierten en ellos. Además, también es un hecho contrastado que la tarea no es sencilla. En el ámbito empresarial a partir de los años sesenta, el modelo de Kirkpatrick (1998), que considera cuatro niveles para evaluar la formación, es uno de los más aceptados. A partir de él se inician investigaciones en las que se profundiza sobre el tercer nivel, el llamado de transferencia de la formación. Baldwin y Ford (1988) ofrecen una definición ampliamente aceptada por expertos y expertas, según la cual transferir consiste en aplicar al lugar de trabajo los conocimientos, habilidades y actitudes adquiridos en un contexto de formación.

Los sistemas directos consisten en medir el grado de transferencia mediante técnicas e instrumentos elaborados y aplicados para cada situación específica de formación. Pruebas como las plantillas de observación de conductas y las pruebas objetivas medirían la transferencia de forma más objetiva, mientras que las entrevistas y los cuestionarios harían lo propio pero de forma más subjetiva, atendiendo a las percepciones de las personas formadas.

Los sistemas indirectos de evaluación de la transferencia se basan en detectar qué variables facilitan o dificultan la aplicación al lugar de trabajo de lo aprendido en la formación. Este tipo de sistemas son menos costosos que los directos, tanto en lo que se refiere al esfuerzo, como en lo referido a lo económico. Varios autores ofrecen modelos para seguir en la evaluación indirecta de la transferencia: Noe (1986); Baldwin i Ford (1988); Burke y Hutchins, (2008), siendo el modelo de Holton (1996; 2005) uno de los más difundidos y aceptados por la comunidad científica. En el contexto español, Pineda, Quesada y Ciraso (2011) crean el primer modelo de factores de la transferencia en el contexto español: el llamado modelo FET (Factores para la Evaluación indirecta de la Transferencia).

La evaluación de la formación docente universitaria es abordada por diversos investigadores como Guskey (2002), Gibb y Coffey (2004), Devlin (2008), Hanbury, Prosser y Rickinson (2008) y Stefani (2011), entre otros. Mayoritariamente la evaluación se refiere solamente a la satisfacción del participante aunque los modelos de Krever y Brook (2001), Guskey (2002) y Zabalza (2011) analizan tanto el impacto de la formación sobre los participantes (en qué han cambiado sus percepciones sobre la docencia, qué han aprendido, etc.), como el impacto en los estudiantes (cómo estos perciben los cambios en la docencia del profesorado a raíz del proceso de formación realizado por este último), como el impacto en la cultura docente de la institución (importancia que se le da a la formación continua, recursos dedicados a la formación, etc.). Todos estos modelos ofrecen posibilidades de evaluación, aunque llevarlas a cabo es una tarea compleja, entre otras razones y centrándonos en la medida de la transferencia, porqué se trata de un procedimiento de medición directa. La evaluación indirecta, pues, aparece como una solución posible al problema de cómo abordar la complejidad de evaluar la transferencia de la formación docente en ámbito universitario.

El "Cuestionario de Transferencia de la Formación Docente" (Feixas, 2013a) contiene 50 ítems agrupados en 8 factores:

○ Diseño de la formación y aprendizaje realizado 


\section{Apoyo del responsable docente \\ Predisposición al cambio \\ Recursos del entorno \\ Feedback del estudiante \\ Reconocimiento institucional \\ Cultura docente del equipo de trabajo y \\ Organización personal del trabajo}

Además existen cada vez más estudios en esta dirección, como los realizados por Pérez y De Corral (2012) en tres promociones de profesorado que ha cursado el Programa de Formación Inicial ProFI del ICE de la UPC, o los de Suárez-Ojeda,Blanch, Cayón, Fuentes, Gimeno y González, (2012) del grupo de innovación docente GI-CAES, interesados en analizar las diversas tipologías de formación implementadas por ellos mismos con el objetivo de valorar la incidencia de la formación recibida por los participantes en su práctica docente. Asimismo Madinabeitia (2014) da cuenta del estudio empírico que aporta evidencias del impacto de la formación en los conceptos de enseñanza y aprendizaje docentes, en su desempeño y también en la capacidad para investigar la docencia o liderar en ámbitos docentes, aspectos propios del cambio organizativo. La investigación llevada a cabo por Acosta, Feixas y Quesada-Pallarès (2016) en las facultades de ingeniería de Chile, abala también la necesidad de comprender como mejorar en los factores relativos a la organización personal del trabajo académico y como incrementar la voluntad para el cambio en el profesorado. El estudio de Díaz Martín (2016) pone de manifiesto la importancia del reconocimiento institucional sobre la labor docente y el esfuerzo del profesorado para mejorar su praxis. Finalmente, Amber y Suárez (2016) añaden que el diseño formativo ha de ser muy focalizado y ha de fomentar el trabajo en colaboración para llegar a ser transformador de la docencia universitaria en el aula.

\section{Metodología}

Como se comentaba anteriormente, una vez obtenidos los resultados relativos a la primera fase del estudio de tipo cuantitativo, se procedió a profundizar en su significado mediante una segunda fase del diseño de tipo cualitativo, siguiendo una metodología integrada (Morgan 2014). Mediante el "focus group" o grupo de discusión, que consiste en realizar una entrevista grupal, se selecciona a un grupo de participantes que están estrechamente relacionados con el tema a tratar, con el objetivo de que hablen sobre dicho tema interactuando entre ellos, de manera que expongan sus vivencias al respecto, sus actitudes y sus opiniones, comentando, a la vez, lo expuesto por sus compañeros (Morgan, 1991).

Se llevaron a cabo 3 grupos de discusión, uno en cada una de las 3 universidades elegidas para el estudio. La duración fue la considerada estándar (Krueger, 1991; Morgan, 1991) entre una hora y media y dos horas y también el número de participantes, que osciló entre 6 y 8 personas. Concretamente en la Universidad 1 participaron 8 personas, en la Universidad 2, participaron 7 y en la Universidad 3, participaron 6 personas, además de la persona moderadora, la misma en todas ellas.

Las personas participantes fueron previamente seleccionadas y posteriormente invitadas a participar en función de su perfil profesional en la universidad: profesores y profesoras relacionados con la formación docente del profesorado. Así, los grupos fueron compuestos por: 
- Coordinadores de Titulación de diferentes Facultades

- Responsables de la Unidad de Formación del Profesorado de las 3 Universidades

- Responsables Institucionales: Vicerrectorado de Calidad, de Ordenación Académica, etc.

- Formadores de docentes experimentados

- Participantes en la formación docente.

El sistema de selección se articuló mediante contacto directo, estableciendo redes topológicas con los participantes, tanto de tipo natural como de tipo artificial, conteniendo relaciones asimétricas (Ibáñez, 1994).

La persona moderadora actuó focalizando el tema a tratar mediante preguntas previamente diseñadas y siguiendo un protocolo definido por el equipo: acogía a su llegada a cada participante, agradeciendo su presencia y procurando su acomodo en una mesa rectangular. Una vez sentados todos los miembros procedía a explicar brevemente la técnica del "focus group" haciendo especial hincapié en sus objetivos, en el papel poco directivo que ejercería la persona moderadora y lo que se esperaba de los participantes: su participación en forma de opiniones, experiencias, ejemplos, comentarios a las aportaciones de los demás compañeros de grupo, etc. Seguidamente exponía el tema de interés del grupo de investigación sobre el que debían tratar los participantes: qué significa transferir lo aprendido en la formación, cuales son los factores que condicionan la transferencia de la formación docente y qué debería cambiarse y cómo para que se incrementara la transferencia de lo aprendido en la formación a la práctica profesional en el aula universitaria.

Una vez clarificado el tema a abordar, quien moderaba explicaba la organización del tiempo, invitando a los participantes a intervenir en una primera ronda siguiendo un orden acordado, y a seguir interviniendo ya sin orden, una vez terminada la primera ronda.

Durante la discusión la persona moderadora mantenía una actitud abierta para captar temas emergentes, no contemplados en el guión de entrevista que pudiesen ser de valor para alcanzar los objetivos del estudio, es decir, para obtener la máxima comprensión sobre las percepciones de los participantes sobre el fenómeno o tema investigado (Morgan, 1991).

Las sesiones fueron registradas mediante video, previo consentimiento de los participantes. Las grabaciones obtenidas fueron transcritas y, a fin de procesar la información y gestionar los datos, se utilizó el programa de análisis cualitativo Atlas Ti 5.0. Este programa informático permitió la organización completa de los datos obtenidos. La información fue cargada en el programa como unidad hermenéutica factores-transferencia. Se procedió a la codificación que consistió, básicamente, en asignar códigos a las unidades de significado y conformar categorías. En la construcción de categorías, se ubicaron distintos códigos dentro de unidades o conceptos teóricos vinculados a la transferencia de la formación. La categorización en sí misma fue una operación conceptual de agrupamiento a partir del análisis de contenido temático. Siguiendo a Massey (2011) en el proceso de análisis de contenido, se han obtenido datos de tipo articulado en su gran mayoría y de tipo emergente en un número menor. 


\section{Resultados}

Presentamos los resultados de estas entrevistas grupales organizados en relación al propio concepto de transferencia, a los facilitadores y las barreras y por último a las propuestas de mejora que han emergido de los propios grupos focales.

\section{Factores que facilitan la transferencia de la formación docente en relación a las personas}

En primer lugar se habla de la motivación del participante para transferir. Los expertos mencionan la motivación varias veces con matices diversos, siempre señalada como un condicionante esencial: "yo trato de transferir algo cuando veo en ello una oportunidad de mejora, pero ojo, que tengo que estar dispuesta emocionalmente, dispuesta a querer trabajar, porque eso no va a ser fácil que lo ponga en práctica ya."(U1/U2)

Se da un tipo de motivación que es intrínseca y que algunos expertos consideran como imprescindible: "La capacidad depende de que quieras o no, porque estamos en un entorno en el cual nos pagan lo mismo por hacer o no hacer. Luego, el hecho de hacer o no hacer, dependerá de que queramos hacer o no[...]". (U1)

Otro factor que repercute en la transferencia de la formación docente es el apoyo de los coordinadores de titulación o responsables docentesy en caso de no tenerse, es un claro obstáculo para la transferencia de la formación. Sin apoyo por parte de coordinadores y responsables de titulaciones, es posible que el curso no tenga ningún impacto. Incluso, a veces "el que quiere hacer una cosa diferente tiene que hacerlo de manera clandestina" $\left(U_{1} / U_{2}\right)$ puesto que la rigidez de las estructuras y de la cultura universitaria hacen de barrera a la transferencia.

La predisposición al cambio de los participantes en los cursos y la que perciben los participantes en su entorno próximo (departamento, titulación, facultad, etc.) es un factor importante para la transferencia. Expertas y expertos hablan de ambos factores indistintamente, lo que parece indicar que la predisposición a cambiar en algo la propia docencia depende en gran medida del apoyo recibido por parte de los responsables que rodean al participante en la formación.

\section{Factores del programa formativo que facilitan la transferencia de la formación docente}

En cuanto al diseño de la formación, surgen cinco temas que influyen en la transferencia: la utilidad percibida, las posibilidades de reflexionar sobre la propia acción docente, la adaptación al contexto de los participantes, que el participante tenga que generar un producto aplicando lo aprendido y la existencia de actividades de seguimiento de la formación.

Respecto la utilidad de la formación, ésta se la considera útil sólo si tiene carácter instrumental y práctico. El carácter práctico se define como: "cercanía con la realidad; aplicación directa al aula; aplicabilidad; usabilidad". (U1/U2)

Otros dos expertos expresan la misma idea así: "la cercanía con tu realidad es fundamental en todo proceso de transferencia. Cuando aquello que comprendes lo percibes cercano, formando parte de tu propia cotidianidad, más fácil lo transfieres. Cuanto más lejano está, más dificultoso es y más necesitas ayuda" ( $\left.\mathrm{U}_{1} / \mathrm{U}_{2}\right)$

Respecto la coherencia entre la actitud y el discurso del formador se consideran elementos relevantes para la eficacia de la transferencia: "pienso que la manera como a veces se hace la formación genera actitudes muy resistentes. Porque, entre lo que decimos y lo que hacemos 
hay contradicción. Por lo tanto, los profesores que vienen a los cursos, reflexionarán sobre lo que hacen y a partir de ahí decidirán si vale la pena o no introducir una cosa diferente". (U3)

Respecto al grado en que el diseño está contextualizado a la realidad del participante el sentir de los expertos es que frecuentemente se cuida más la calidad de los contenidos formativos que las posibilidades que tendrán los participantes de aplicar lo aprendido a su docencia y ello es un error. Así, lo adecuado sería que se plantease la formación desde el conocimiento de lo que hace el profesor que acude a la formación. El diseño formativo debería estar adaptado al contexto real de los participantes en la formación: "tú te apuntas en un curso porque piensas que aquello lo podrías utilizar y vas allá, te explican las cosas y dices no, es muy complicado(...). Claro que si me explican cosas que me dan ideas que sí, intentaré colocarlo en mi asignatura o en el grupo de profesores". (U3)

El hecho de generar algún producto utilizando lo aprendido parece ser un factor importante. No basta con asistir al curso, sino que es necesario integrar en la asignatura o en la práctica docente lo aprendido en la formación: "Hace unos años, lo normal era hacer un curso y luego se daba una certificación. Pero yo creo que eso es una equivocación, puesto que hasta que ellos no lo lleven al aula integran bien lo aprendido. Por lo que cambiamos de estrategia, y prescindimos de cursos en los que se enseñaba algo y nada más, al tipo de curso+ producto". (U1/U2).

Complementario a ello el diseño de actividades de seguimiento, una vez la formación presencial ha concluido, facilita dicha transferencia. Se citan diversas modalidades: tutorías entre iguales; tutorías grupales: foros de intercambio y reflexión: y asesorías.

La finalidad del seguimiento es superar las dificultades propias en la implementación. Parece que, como afirmaban los expertos, el seguimiento a través de las nuevas tecnologías es un camino abierto.

\section{Factores organizacionales que facilitan la transferencia}

El profesorado que asiste a los cursos pertenece a unidades organizativas en las cuáles hay una estructura que influye y determina las acciones que éste haga.

El departamento es una de estas unidades organizativas. Se considera que es "una estructura del siglo XVIII, está escondida y nos puede generar problemas [‥] es una estructura que en muchos casos se utiliza para mantener una jerarquía de decisiones" ( $\mathrm{U}_{1} / \mathrm{U}_{2}$ ) y dicha estructura choca con la necesidad de crear equipos docentes que funcionen independientemente de la procedencia departamental de sus miembros.

Contar con el beneplácito de la dirección departamental es un requisito que se considera necesario para transferir: "Yo pienso que es muy importante que el jefe de departamento pueda hacer equipos predispuestos, porque si no, no tiene ningún sentido. Es decir, como mínimo el jefe de departamento tienen que dejar hacer". (U3)

La coordinación entre el profesorado de las asignaturas es otro elemento que facilita la transferencia: "yo supongo que las asignaturas donde hay varios profesores implicados, sí que hay una coordinación clara y trabajan con los mismos objetivos. Y luego lo que parece más complicado es plantear esa coordinación a nivel de departamentos, eso es ya más dificultoso". (UPV).

El reconocimiento al esfuerzo que hacen los docentes para aplicar lo aprendido en la formación facilita la transferencia de éste. Se pueden diferenciar dos ámbitos de reconocimiento: el más ligado a la institución a la que se pertenece y el asociado a la comunidad científica relativo al desarrollo de la carrera académica.

El apoyo entre iguales se considera un elemento facilitador de la transferencia: "creo que 
para que la formación docente verdaderamente sea transferible tienes que tener apoyo sea institucional o entre compañeros" (U3).

Finalmente, remarcar que se considera un ideal deseable una cultura colaborativa: "hay algunas cosas que quisiera hacer, que si yo tuviese la seguridad de que alguien me ayudaría, sería fácil y lo haría". (U3)

\section{Barreras que impiden la transferencia de la formación docente}

La falta de motivación es una barrera clara para la transferencia. La desmotivación puede venir por distintas razones, una de ellas tiene que ver con las dificultades que se encuentra el profesorado una vez ha terminado la formación y ha de introducir cambios en el día a día: "nos cuesta motivarnos, los mismos profesores que vienen a los cursos te dicen que es súper interesante y salen motivados pero claro cuando van al aula ven que les falta recursos". $\left(U_{1} / U_{2}\right)$

Otro elemento que dificulta la motivación son las perspectivas laborales que tiene el profesorado universitario actualmente: "tal como tiene las perspectivas el profesorado, no hay nada que los motive y para mi es el problema de base actual. $Y$ esto produce un desencantamiento. (U3)

Un obstáculo que se señala en relación a la estructura es cómo hacer llegar la innovación al catedrático, y/o al profesorado consolidado que no ve la necesidad de cambiar nada y puede sentir amenazada su posición. Algunos expertos consideran que si no se implican las personas de más rango académico, difícilmente lo harán las de menor rango. Sin embargo, otros expertos creen lo contrario, que si los que introducen cambios son los de menor rango, las posibilidades de que el profesorado consolidado se anime a cambiar, se incrementan: "En este sentido, pienso que explicarle a un becario cómo hacer cosas nuevas es una muy buena manera de que el catedrático organice sus tareas. Y es mucho más efectivo que el becario le diga al catedrático [propuestas de acción] que no que el jefe de departamento diga: a partir de ahora todos los profesores han de hacer las cosas de esta manera". (U3)

Los recursos con los que cuenta la persona docente para aplicar lo aprendido también representan un obstáculo aunque los y las expertas no le dan gran importancia, de hecho comentan: "los aspectos físicos son un condicionante clarísimo, pero para todo tipo de enseñanza" ( $\left.U_{1} / U_{2}\right)$. Por ello, se considera que los impedimentos para la transferencia no son de tipo material, sino de tipo más intangible.

El poco o negativo feedback que reciben los docentes de los estudiantes sobre su praxis docente es insuficiente en los modelos de evaluación de la docencia que se aplican actualmente, de corte cuantitativo. Sería necesario cambiar el modelo de evaluación que se les pide a los alumnos: "Es difícil saber qué es el éxito docente pero tiene que ver con la percepción del profesor de que ha tenido éxito con su proceso educativo. Eso es complicado de establecer porque hay una tradición muy larga de evaluación con encuestas". (UPV)

El reconocimiento académico es percibido como necesario e insuficiente, en tanto que en la actualidad se valora mucho más la tarea investigadora que la tarea docente. Así, la formación para la función docente será tenida en cuenta: "en tanto y cuanto eso sirva como mérito para estabilizar tu acreditación, es decir, y en tanto y cuanto no lo sea, seguiremos actuando desde el querer. Pero creo que ahí se necesita un cambio". (U1/U2)

La falta de reconocimiento de la docencia es vista como una barrera, se considera que el modelo actual premia la investigación para "liberarte de ser profesor" ( $\left.U_{1} / U_{2}\right)$, esa mentalidad "choca de frente con toda la intención de la formación docente" (U1/U2), puesto que "el más listo es el que menos docencia da". (U1/U2) 
El reconocimiento institucional se relaciona con el apoyo percibido por parte del responsable docente y con las resistencias halladas para la transferencia en el entorno inmediato del profesorado: "el reconocimiento no está en el currículum y lo que has hecho, sino que si todos son conscientes, ya es gratificante [...] yo me centraría en el apoyo y en la concienciación. El reconocimiento es que la gente sepa que se están transfiriendo aprendizajes al aula". (U3)

La cultura docente del trabajo en equipo es otro de los factores considerados importantes para la transferencia de lo aprendido. Los expertos consultados entienden que cada vez más se necesita del grupo para poder funcionar: "ahora ya no solo sirve aprender algo y llevarlo a mi aula, sino que ahora lo tengo que compartir con los demás" (U1/U2)

El problema es que la cultura universitaria establecida a menudo es una barrera para el trabajo en equipo. La cultura en muchas ocasiones aparece caracterizada como: "parcelación del trabajo, la falta de cultura colaborativa" (U1/U2), donde el profesorado se queda con la interpretación de la ley o de la norma que le interesa a cierto grupo de profesores: "para seguir haciendo lo que le da la gana" (U1). Hay transferencia que se deja de hacer por la falta de reconocimiento y de valoración del contexto y su cultura organizativa.

Finalmente, el último factor considerado, consiste en la organización personal del trabajo. Las personas expertas mencionan unos cuantos obstáculos al respecto. En primer lugar aluden al tiempo que el profesorado no estable tiene que dedicar a los procesos de acreditación: "la queja más general es que no tienen tiempo para nada. Tienen que investigar, tienen que sacar artículos..." ( $\left.U_{1} / U_{2}\right)$

La estabilidad es vista de dos maneras: como facilitadora de la transferencia, pues da margen y seguridad de que el esfuerzo invertido tendrá visión de futuro. $\mathrm{Y}$, por otro lado, "si fuese verdad que la estabilidad es favorecedora de innovación todos los catedráticos y titulares lo serían". (U3)

Parece que en la priorización de tareas, las relativas a la investigación son percibidas como más importantes que las relacionadas con la docencia, entre otros motivos porque los resultados son más visibles: "la estrategia de la investigación tiene unas consecuencias efectivas mucho más realistas que el esfuerzo docente" (U1); "el buen docente, en general, es menos visible que el maravilloso investigador que es más conocido. Entonces está claro que hay una cosa que el sistema no quiere ver, o no le apetece, o no sabe cómo resolverlo" (U1).

\section{Propuestas para incrementar la transferencia de la formación docente}

Una propuesta mencionada reiteradamente por las personas expertas es que el diseño formativo no acabe en el aula, sino que se extienda en el tiempo y en el espacio más allá de ella. Los responsables de la formación podrían hacer el seguimiento después de la formación y ello favorecería la transferencia. Las nuevas tecnologías son un medio considerado excelente para ello: "Esto puede ir acompañado de un equipo virtual, que una vez que ha acabado eso, entran en una siguiente fase y tengo opción de comentar mis dudas, mis inseguridades, mis certezas. Eso sí ayuda también a la transferencia". (U1/U2)

Contextualizar los contenidos de la formación a la realidad próxima de los participantes, es otra de las propuestas que surgen: "muchas veces si la gente no ve un ejemplo concreto en su área de conocimiento en casos prácticos de éxito o fracaso no los motiva. Puede ser que si hicieran una jornada de discusión y reflexión..." (U3); "La cercanía con tu realidad es fundamental en todo proceso de transferencia. Cuando aquello que comprendes más cercano está de tu propia cotidianidad, más fácil lo transfieres. Cuanto más lejano está, más dificultoso es y más necesitas ayuda". (U1/U2)

Se considera que el hecho de pedir un producto final fruto de la aplicación de lo aprendido 
en la formación también juega a favor de la transferencia: "las actividades de seguimiento, es decir, que haya un pequeño producto relacionado con la temática del curso. Por ejemplo, hemos dado evaluación de competencias, pues ahora diseñaremos una prueba de evaluación, que la corrige el propio formador y están trabajando temas propios de su práctica". (U1)

La orientación de la formación hacia la reflexión sobre la propia práctica, también es una propuesta compartida por muchos de los expertos: "creo que hace falta la reflexión sobre qué es lo que sale bien y por qué sale bien y lo que no. Yo creo que esto es más eficiente que mirar de hacer cosas muy diferentes. Que también llegan estas cosas diferentes, estos cambios son inherentes a la reflexión" (U3); "para transferir los conocimientos docentes, se han de hacer unas labores de concienciación para que el profesor sepa lo que le falta, porque muchos no son conscientes de sus carencias. Saben que no saben, pero no tienen una buena idea de que es lo que no saben. Porque si lo supieran, supongo que ellos suplirían esa carencia". (U1)

Finalmente, diversificar la oferta formativa también es una propuesta que se lanza, para conseguir, fundamentalmente, que el profesorado que no participa frecuentemente en la formación, lo haga y pueda, así, transferir lo aprendido y generar cambios en el aula: "los profesores ya veteranos no suelen venir, esto no quiere decir que no hagan actividad de formación, ... "(U1)

Podríamos sintetizar las aportaciones de los participantes en estos tres grupos de discusión en la siguiente tabla:

Tabla 1.

Factores facilitadores, barreras y propuestas para la transferencia de la formación docente.

\begin{tabular}{|c|c|c|}
\hline $\begin{array}{l}\text { Factores que facilitan la } \\
\text { transferencia }\end{array}$ & $\begin{array}{l}\text { Barreras que impiden } \\
\text { la transferencia }\end{array}$ & $\begin{array}{l}\text { Propuestas para mejorar } \\
\text { transferencia }\end{array}$ \\
\hline $\begin{aligned} & \text { Personales }> \\
& \text { la motivación } \\
& \text { intrínseca } \\
& \text { la predisposición al } \\
& \text { cambio } \\
& \text { Del programa formativo }> \\
&> \text { la utilidad, } \\
&> \text { Contextualización, } \\
&> \text { Generar un producto } \\
& \text { al finalizar la } \\
& \text { formación } \\
& \text { Organizacionales } \\
&>\text { El apoyo de los } \\
& \text { coordinadores o } \\
& \text { responsables } \\
& \text { docentes, } \\
&> \text { El beneplácito de la } \\
& \text { Dirección } \\
& \text { Departamental } \\
&> \text { La coordinación entre } \\
& \text { profesorado, } \\
&> \text { El reconocimiento al } \\
& \text { esfuerzo } \\
&> \text { El apoyo entre iguales }\end{aligned}$ & $\begin{array}{ll}\text { - } & \text { Recursos } \\
\text { insuficientes, } \\
\text { - Poco feedback } \\
\text { por parte del } \\
\text { estudiantado, } \\
\text { - Poco } \\
\text { reconocimient } \\
\text { o académico } \\
\text { de la docencia } \\
\text { y } \\
\text { Cultura de } \\
\text { equipo } \\
\text { docente } \\
\text { insuficiente }\end{array}$ & $\begin{array}{l}\text { Diseño del programa } \\
\text { formativo que incluya } \\
\text { seguimiento en el } \\
\text { tiempo, } \\
\text { - Contextualizar los } \\
\text { contenidos, } \\
\text { Orientar el programa } \\
\text { hacia la autorreflexión } \\
\text { de la propia práctica y } \\
\text { Diversificar las } \\
\text { propuestas formativas }\end{array}$ \\
\hline
\end{tabular}




\section{Conclusiones}

La evaluación de la transferencia de la formación en la universidad se estima como un elemento clave para incidir en el desarrollo docente. Por ello, el estudio que presentamos contribuye a identificar los factores clave que se deben incluir en cualquier proceso evaluativo de la transferencia.

Se espera que la formación docente promueva un cambio en las habilidades, conocimientos y concepciones del profesorado participante (Maríny Teruel, 2004) y que éste lo aprenda e interiorice para que, en su contexto particular, pueda aplicarlo de manera efectiva y sostenida.

Existen un conjunto de factores que pueden estimular o inhibir la transferencia a la práctica y éstos pueden variar de individuo a individuo, dependiendo del propósito y del entorno y condicionar el éxito de los programas de formación. Las estrategias formativas difieren mucho entre sí y para realizar una evaluación ajustada debemos conocer qué funciona, para quien y bajo qué circunstancias. Es decir, debemos conocer los factores que afectan e influyen en la transferencia del aprendizaje de los docentes. La discusión analizada entre los miembros expertos en formación docente universitaria de las tres universidades revela la existencia de factores individuales, factores relativos al programa y factores institucionales que determinan e influyen en la calidad y eficacia de lo aprendido en la formación realizada.

A su vez se han deducido una serie de factores condicionantes de esta transferencia que se pueden clasificar en facilitadores y en barreras. Fruto de este análisis de factores emergen una serie de propuestas de mejora tales como:

- El diseño formativo no debe acabar en el aula, sino que se debe extender en el tiempo más allá de ella, es decir hacer un seguimiento después de la formación proporcionada.

- Contextualizar los contenidos de la formación a la realidad próxima de los participantes.

- El programa formativo debe incorporar la elaboración de un producto final por parte del profesorado asistente para que éste vea aplicable lo aprendido.

- Fomentar una cultura de trabajo en equipo entre el profesorado a fin que la transferencia contribuya a una cultura colaborativa y

- Contribuir a elevar el reconocimiento de la tarea docente y equipararla a la tarea investigadora.

Con todo, no podemos hacer una interpretación general de las políticas de desarrollo docente que se están llevando a cabo en la actualidad, aunque tampoco era éste el objetivo del estudio, pero sí mostrar la predominancia de ciertos condicionantes de la transferencia de la formación docente recibida por nuestros docentes, coincidentes con los análisis de la literatura más actual (Blume et al., 2010 y De Rijdt et al., 2013). Finalmente, la herramienta ha demostrado su consistencia y validez y ello nos permite seguir explorando nuevos contextos formativos y analizando factores, tanto de forma integrada como factores concretos. Futuras investigaciones sugieren explorar más a fondo de qué manera los factores del entorno (la cultura docente del equipo de trabajo o el rol del responsable docente, por ejemplo) facilitan la transferencia de los aprendizajes realizados en el contexto laboral del profesor universitario. 


\section{Referencias}

Acosta Peña, R.; Feixas, M. \& Quesada-Pallarés, C. (2016). From discourse to action. ¿How Engineering' university teachers in Chile develop professionally and transfer their learning into practice?ZeitschriftfürHochschulentwicklung (ZFHE), 11(5), 163-185.

Baldwin, T. T., \& Ford, J. K. (1988). Transfer of training: A review and directions for future research. Personnel Psychology,41(1), 63-105.

Blume, B. D., Ford, J. K., Baldwin, T. T., \& Huang, J. L. (2010). Transfer of training: A meta analytic review. Journal of Management, 36(4), 1065-1105

Burke, L.A., \& Hutchins, H.M. (2008). A study of best practices in training transfer and proposed model of transfer. Human Resource Development Quarterly, 19(2), 107-128.

De Rijdt, C., Stes, A., Van der Vleuten, C., \& Dochy, F. (2013). Influencing variables and moderators of transfer of learning to the workplace within the area of staff development in higher education: research review. Educational Research Review, 8, 48-74.

Devlin, M. (2008). Research challenges inherent in determining improvement in university teaching. Issues in Educational Research, 18(1). http://www.iier.org.au/iierl8/devlin.pdf

Díaz Martín, W. (2016). Formación del profesorado universitario, evaluación de la actividad docente, recursos y promoción profesional. Estudios Pedagógicos XLII, 1, 65-85.

Feixas, M., Duran, M. M., Fernández, I., Fernández, A., García San Pedro, M. J., Márquez, M. D., Pineda, P., Quesada, C., Sabaté, S., Tomàs, M., Zellweger, F. \& Lagos, P. (2013 a). ¿Cómo medir la transferencia de la formación en Educación Superior?: el Cuestionario de Factores de Transferencia. Revista de Docencia Universitaria, 11(3), 219-248.

Feixas, M., Fernández, A.Lagos,P., Quesada, C y Sabaté,S.(2013b). Factores condicionantes de la transferencia de la formación docente en la universidad: un estudio sobre la transferencia de las competencias docentes. Infancia y Aprendizaje: Journal for the Study of Educacion and Development, 36 (3), 401-416.

Gibbs, G. \& Coffey, M. (2004). The impact of training of university teachers on their teaching skills, their approach to teaching and the approach to learning of their students. Active Learning in Higher Education, 5(1), 87-100.

Guskey, T. (2002). Does it make a difference? Evaluating professional development. Educational Leadership, 59(6), 45-51.

Hanbury, A., Prosser, M. \& Rickinson, M. (2008). The differential impact of UK accredited teaching development programmes on academics' approaches to teaching. Studies in Higher Education, 33(4), 469-483.

Holton, E. F., III. (2005). Holton's evaluation model: New evidence and construct elaborations. Advances in Developing Human Resources, 7 (1), 37-54.

Holton, E.F. (1996). The flawed four-level evaluation model. Human Resources Development Quarterly, 7, 5-21.

Ibáñez, (1994) en Kirkpatrick D. L. (1998). Evaluating training programs. The four levels. (2nd edition). San Francisco: Berrett-Koehler Publishers, Inc.

Kreber, C. \& Brook, P. (2001). Impact evaluation of educational development programmes. International Journal for Academic Development, 6(2), 96-108.

Madinabeitia,A.(2014). Impacto global del desarrollo docente en la universidad.El caso del programa ERAGIN en la Universidad del País Vasco (UPV/EHU). Revista del CIDUI. CIDUI's Journal, 2. 
Marín, M y Teruel, M.P. (2004).La formación del docente universitario: Necesidades y demandas desde su alumnadoRevista Interuniversitaria de Formación del Profesorado, 18(2), 137-151.

Montes, D. A. y Suárez, C. I. (2016). La formación docente universitaria: claves formativas de universidades españolas.Revista Electrónica de Investigación Educativa, 18(3), 51-64.

Noe, R.A. (1986). Trainees' attributes and attitudes: Neglected influences on training efficacy. Academy of Management Review, 11, 736-49.

Pérez,M.J. y De Corral, I (2012). L'avaluació de la transferència i l'impacte de la formació inicial del professorat a la UPC.Revista del CIDUI. CIDUI's Journal, 1.

Pineda, P., Quesada, C., \& Ciraso, A. (2011). Evaluating Training Effectiveness: results of the FET Model in the Public Administration in Spain. Actas del 7th International Conference on Researching Work and Learning (Shanghai, Dec 4-7, 2011).

Stefani, L. (Ed.) (2011). Evaluating the effectiveness of academic development: Principles and practice. New York: Routledge.

Suárez-Ojeda,M.E, Blanch,S., Cayón,M., Fuentes,M., Gimeno,X. y González,N.(2012). Análisis de la tipología de la formación permanente del profesorado universitario para el uso de carpetas de aprendizaje en la educación superior en la universitat autònoma de barcelona.Revista del CIDUI. CIDUI's Journal, 1.

Zabalza, M.A. (2011). Evaluación de los planes de formación docente de las universidades. Educar, 47, 181-197. 